\title{
Die Markgrafen von Baden im Gebiet von Neckar und Murr (11. bis 13. Jahrhundert)
}

\author{
Von Heinz Krieg
}

\section{Zur Geschichte der frühen Markgrafen von Baden}

Am Anfang der Reihe der Markgrafen von Baden steht mit Hermann I. ein Markgraf, der streng genommen noch gar nicht als Markgraf von Baden anzusprechen ist. Denn Hermann I. war tatsächlich ein Markgraf von Verona ${ }^{1}$ (Abb. 1). Trotzdem wurde Markgraf Hermann I. als Spitzenahn des Hauses Baden angesehen, obwohl eigentlich erst sein gleichnamiger Sohn, Hermann II., nach dem Ort Baden zubenannt wurde. Diese Erstnennung in einer in zweifacher Ausfertigung überlieferten Urkunde Kaiser Heinrichs V. vom 27. April 1112 lieferte auch den zeitlichen Anknüpfungspunkt für die Karlsruher Ausstellung zum 900-jährigen Jubiläum Badens².

Eine ähnliche ,Vereinnahmung' wie sie Hermann I. von Seiten seiner Nachkommen, eben der Markgrafen von Baden, erfuhr, lässt sich im Übrigen auch bei den mit den markgräflichen Hermannen verwandten Herzögen von Zähringen beobachten. Denn auch Herzog Bertold I., der Vater Hermanns I. und Bertolds II., war selbst eigentlich noch kein Zähringer. Vielmehr wurde erst Herzog Bertold II. zum Begründer des Geschlechts der Zähringer, indem er die im nördlichen Breisgau gelegene Burg Zähringen (heute Stadtteil von Freiburg) bezog, die für das zähringische Herzogsgeschlecht namengebend werden sollte ${ }^{3}$. Sein gleichnamiger Vater

1 Siehe auch zum Folgenden Heinz Krieg: Baden. In: Fürstliche Höfe und Residenzen im spätmittelalterlichen Reich. Ein dynastisch-topographisches Handbuch. Hg. von Werner Paravicini, Jan Hirschbiegel und Jörg Wettlaufer (Residenzenforschung 15.I). Ostfildern 2003. Bd. 1. S. 37. - Hansmartin Schwarzmaier: Hermann I. von Baden. In: LexMA 4. München/Zürich 1989. Sp. 2160. - Karl Schmid: Baden-Baden und die Anfänge der Markgrafen von Baden. In: ZGO 140 (1992) S. 1-37.

${ }^{2}$ Heinz Krieg: Die Ersterwähnung Badens 1112. In: Baden! 900 Jahre. Geschichten eines Landes. Hg. von Oliver Sänger. Karlsruhe 2012. S. $26 \mathrm{f}$.

${ }^{3}$ Siehe (mit weiterer Literatur) ders.: Adel in Schwaben: Die Staufer und die Zähringer. In: Grafen, Herzöge, Könige. Der Aufstieg der frühen Staufer und das Reich (1079-1152). Hg. von Hubertus Seibert und Jürgen Dendorfer (Mittelalter-Forschungen 18). Ostfildern 2005. S. 65-97. Hier S. 89-29. 
Bertold I. († 1078) Hg. v. Kärnten

$\infty_{1}$ Richwara, T. Hg. Hermanns IV. v. Schwaben?

Hermann I. $(† 1074)$

Gf. im Breisgau, Mkgf. v. Verona

Mönch in Cluny

$\infty$ Judith († 1091)
Bertold II. († 1111)

Hg. v. Zähringen

$\infty$ Agnes, T. Rudolfs v. Rheinf.

Herzöge von Zäbringen

Hermann II. († zw. 1123 u. 1130)

Gf. im Breisgau, im Ufgau, Mkgf. v. Limburg, v. Baden

$\infty$ Judith († zw. 1116 u. 1122)

Hermann III. (†1160 oder n. 1164?)

Mkgf. v. Baden, v. Verona

$\infty$ Berta

Hermann IV. $(\dagger 1190)$

Mkgf. v. Baden, v. Verona

$\infty$ Udelhild

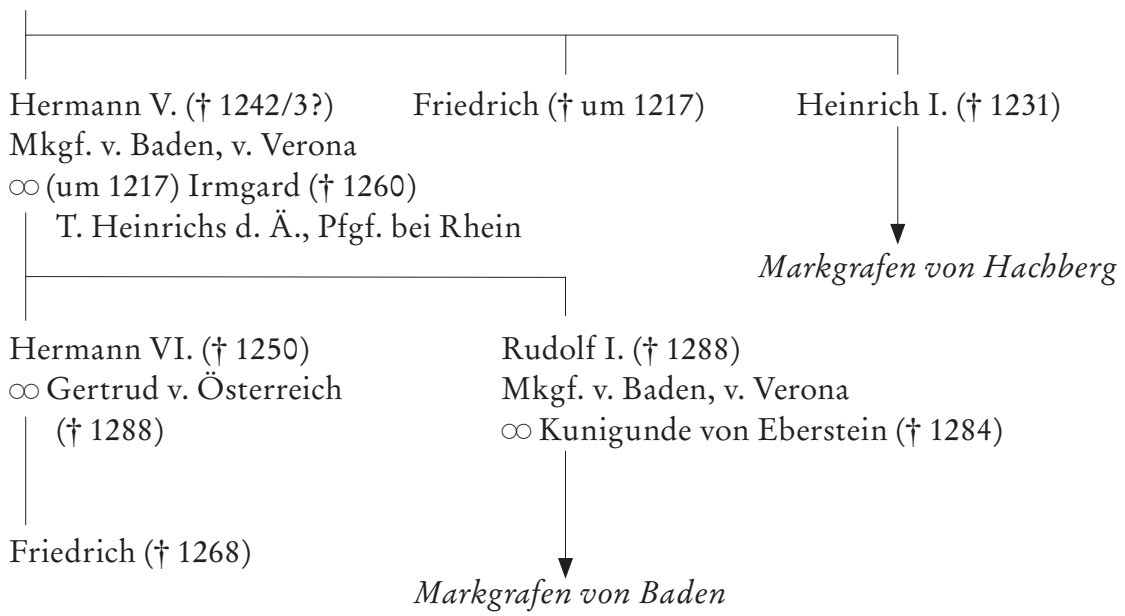

Abb.1: Stammtafel zu den Markgrafen von Baden. 
dagegen hatte seinen Herrschaftsschwerpunkt noch nicht am südlichen Oberrhein im Umfeld der Burg Zähringen, sondern vielmehr im mittleren Neckargebiet auf der Limburg bei Weilheim, wo er nach der Darstellung des Bamberger Chronisten Frutolf von Michelsberg angesichts der von seinem Gegner, dem Salier Heinrich IV., in Schwaben angerichteten Verheerungen wahnsinnig wurde und bald darauf im Jahre 1078 verstarb ${ }^{4}$.

Herzog Bertold I. von Kärnten hatte Hermann I., seinen ältesten Sohn, für sich offenbar als Nachfolger vorgesehen und ihn dementsprechend allem Anschein nach bereits im Vorgriff als künftigen Herzog von Schwaben "aufgebaut“ ${ }^{5}$. Das Herzogtum Schwaben war Bertold I. nämlich von Kaiser Heinrich III. versprochen worden. Doch wider Erwarten blieb es letztlich bei diesem Versprechen, und Bertold konnte nach dem Tod Heinrichs III. seinen Anspruch auf Schwaben nicht durchsetzen. Stattdessen sah er sich gezwungen, dem Grafen Rudolf von Rheinfelden in Bezug auf die schwäbische Herzogswürde den Vortritt zu lassen. Schon zuvor aber hatte Bertold seinen ältesten Sohn auf den traditionsreichen schwäbischen Herzogsnamen Hermann taufen lassen. Doch statt des eigentlich erstrebten Herzogtums Schwaben erhielt Hermanns Vater gewissermaßen als Entschädigung im Jahr 1061 das Herzogtum Kärnten ${ }^{6}$. Danach firmierte sein Sohn Hermann dann als Markgraf von Verona, denn die Mark Verona gehörte damals zum Herzogtum Kärnten. Eine wirkliche Amtwaltung in der Mark Verona ist aber zumindest für Markgraf Hermann I. trotz seines stolzen Titels nicht belegbar.

Nachweislich amtierte Hermann I. jedoch als Graf im Breisgau und trat so als Inhaber jenes Amtes hervor, das schon sein Vater Bertold I. und darüber hinaus auch schon dessen gleichnamiger Vater bekleidet hatten ${ }^{7}$. Die Weichen für Hermanns weitere Karriere als Erbe und Nachfolger seines Vaters waren demnach schon gestellt, und sein weiterer Weg schien klar vorgezeichnet, als Hermann noch als junger Mann im Jahr 1073 der Welt den Rücken kehrte und sich in das burgundische Reformkloster Cluny zurückzog, wo er bereits im darauffolgenden Jahr 1074 verstorben ist. Durch diesen spektakulären Akt der Konversion gewann der

${ }^{4}$ Frutolf von Michelsberg: Chronik. In: Frutolfs und Ekkehards Chroniken und die anonyme Kaiserchronik. Hg. von Franz-Josef Schmale und Irene Schmale-Ott (Ausgewählte Quellen zur deutschen Geschichte des Mittelalters 15). Darmstadt 1972. S. 47-121. Hier S. 88 Z. 24-32. - Siehe dazu Ulrich Parlow: Die Zähringer. Kommentierte Quellendokumentation zu einem südwestdeutschen Herzogsgeschlecht des hohen Mittelalters (VKgL A 50). Stuttgart 1999. Nr. 93. S. 61 f. - Karl Schmid: Sasbach und Limburg. Zur Identifizierung zweier mittelalterlicher Plätze. In: ZGO 137 (1989) S.33-63. - Nach ders.: Vom Werdegang des badischen Markgrafengeschlechtes. In: ZGO 139 (1991) S. 45-77 müssten Herzog Bertold von Kärnten und sein Sohn Markgraf Hermann von Verona, „wenn die beiden schon näher charakterisiert werden sollen“, am ehesten noch als „Limburger" bezeichnet werden, aber man dürfe sie eben nicht als Zähringer oder Badener ansprechen. Siehe dazu auch unten S. 20-23.

${ }^{5}$ Vgl. zum Folgenden Krieg, Adel (wie Anm.3) S.74, 80-82. - Parlow (wie Anm.4) Nr. 27 f. S. 19-21.

${ }^{6}$ Siehe dazu und zum Folgenden Parlow (wie Anm. 4) Nr. 31 S. 22-24.

7 Vgl. Krieg, Adel (wie Anm. 3) S. 69-74. - Parlow (wie Anm. 4) Nr. 47. 
auf diese Weise vom Veroneser Markgrafen zum Mönch mutierte Ahnherr des Hauses Baden zumindest unter den geistlichen Reformern der Zeit des Investiturstreits, aber auch darüber hinaus einige Berühmtheit ${ }^{8}$.

Während die von Vertretern der Kirchenreform geprägte Chronistik der Zeit des Investiturstreits den Ruhm des heiligmäßigen Lebenswandels Markgraf Hermanns I. verbreitete, darf man annehmen, dass für Hermann II., der als noch unmündiges Kind seinen Vater verloren hatte, das politisch wichtigste Vermächtnis seines Vaters weniger dessen Ruf der Heiligkeit als vielmehr der Anspruch auf die Markgrafenwürde war. Der Markgrafentitel, an dem alle Badener über sämtliche hoch- und spätmittelalterlichen Linientrennungen hinweg beharrlich festgehalten haben, stellte sozusagen einen ideellen Trumpf dar und bot entscheidendes Potential. Denn er ermöglichte es, schon in der Titulatur gegenüber den nichtfürstlichen, hochadligen Standesgenossen einen gewissen Vorrang zu demonstrieren und damit den Anspruch auf eine fürstliche Rangstellung zu behaupten?.

Und tatsächlich erlangten die Markgrafen von Baden im hohen Mittelalter unter den letzten Saliern und vor allem unter den Staufern im treuen Dienst am Königshof eine fürstliche Rangstellung. Sie gehörten damit in dieser Zeit ganz sicher zu den bedeutendsten Hochadelsfamilien im Gebiet des ehemaligen Herzogtums Schwaben neben den Württembergern, denen sie rangmäßig zunächst durchaus überlegen waren. Dies lässt sich deutlich in den Zeugenreihen der salischen und staufischen Herrscherurkunden ablesen, in denen die Markgrafen von Baden regel-

${ }^{8}$ So verfasste etwa der Cluniazensermönch Ulrich von Zell, der später zur Ehre der Altäre erhoben wurde, eine verlorengegangene Vita Markgraf Hermanns, vor allem aber fand die bald legendenhaft ausgeschmückte Geschichte des in Cluny als Schweinehirt dienenden Markgrafen Hermann in der zeitgenössischen Historiographie weitere Verbreitung. Siehe Karl Schmid: Vom Werdegang des badischen Markgrafengeschlechtes. In: ZGO 139 (1991) S. 45-77. Hier S. 50, 71-77. - Joachim Wollasch: Markgraf Hermann und Bischof Gebhard III. von Konstanz. Die Zähringer und die Reform der Kirche. In: Die Zähringer in der Kirche des 11. und 12. Jahrhunderts. Hg. von Karl Suso Frank. Freiburg i. Br. 1987. S. 27-53. Hier S. 29-31.

${ }^{9}$ Siehe dazu Heinz Krieg: Die Markgrafen von Baden: Eine Familie am unteren Rand des Fürstenstandes. In: Princely Rank in Late Medieval Europe. Trodden Paths and Promising Avenues. Hg. von Thorsten Huthwelker, Jörg Peltzer und Maximilian Wemhöner (RANK. Politisch-soziale Ordnungen im mittelalterlichen Europa 1). Ostfildern 2011. S.309-332. Hier S. 311. - Ders.: Zur Herrschaftsbildung der Markgrafen von Baden im späten Mittelalter. In: Das Land am mittleren Neckar zwischen Baden und Württemberg. Hg. von Hansmartin Schwarzmaier und Peter Rückert (Oberrheinische Studien 24). Ostfildern 2005. S. 163-187. Hier S. 170 f. - Werner Rösener: Ministerialität, Vasallität und niederadelige Ritterschaft im Herrschaftsbereich der Markgrafen von Baden vom 11. bis zum 14. Jahrhundert. In: Herrschaft und Stand. Untersuchungen zur Sozialgeschichte im 13. Jahrhundert. Hg. von Josef Fleckenstein (Veröffentlichungen des Max-Planck-Instituts für Geschichte 51). Göttingen ${ }^{2}$ 1979. S. 40-91. Nach ebd. S. 43 bewirkte der Titel zwar „keine wirkliche Markgrafenstellung“. Rösener unterstreicht aber, dass „die rechtssymbolische Bedeutung der Titulatur ... nicht unterschätzt werden“ dürfe, „da sie dem Geschlecht ein ideelles Übergewicht über seine Standesgenossen verschaffen konnte“. 
mäßig vor den Grafen rangieren ${ }^{10}$. Die Herrscherurkunden bieten überhaupt die wichtigsten Zeugnisse zu den frühen Markgrafen, denn letztere sind während des 12. Jahrhunderts in erster Linie als Zeugen in den Diplomen der Salier und der Staufer fassbar. Diese Zeugnisse markieren dabei zugleich die enge Anlehnung der Markgrafen von Baden an das Königtum, die für die mindermächtigen Markgrafen von Baden stets von grundlegender Bedeutung bleiben sollte ${ }^{11}$.

Markgraf Hermann I. von Verona hinterließ seinen Nachkommen nicht nur den Anspruch auf die Markgrafenwürde, sondern als weiteres wichtiges Erbe nicht zuletzt auch seinen Namen. Davon zeugt die eindrucksvolle Reihe der Hermanne des 12. bis zur Mitte des 13. Jahrhunderts (vgl. Abb. 1). Die fast hermetisch anmutende Fixierung auf den Leitnamen Hermann darf als eindrücklichstes Zeichen des Selbstbewusstseins der Familie der frühen Markgrafen von Baden gelten. Die Weitergabe des Namens vom Vater auf den Sohn ist hier als Ausdruck der Kontinuität des Geschlechts insgesamt zu verstehen. Dass bei der Nachbenennung nach dem Vater ein Name das ganze Geschlecht repräsentierte, lenkt den Blick zugleich auch auf den ersten Träger dieses Namens, nämlich auf Hermann I. Die Frage, warum sich sein Name offensichtlich über Generationen hinweg bei den markgräflichen Hermannen ungebrochener Attraktivität erfreute, führt aber noch weiter. Denn in diesem Zusammenhang ist an Hermanns Großvater zu erinnern, bei dem es sich aller Wahrscheinlichkeit nach um Herzog Hermann IV. von Schwaben handelte ${ }^{12}$. Das eigentlich Prestigeträchtige am Namen Hermann war also möglicherweise zunächst vor allem die Tatsache, dass Hermann ein gebräuchlicher Name schwäbischer Herzöge war. Das Festhalten der frühen Markgrafen an diesem Namen könnte also darauf hindeuten, dass die markgräflichen Hermanne mit ihrem Leitnamen noch die Erinnerung an ihre hochrangige Abstammung von einem Schwabenherzog beziehungsweise den Anspruch auf einen herzogsgleichen Rang verbanden - dies im Übrigen in einer Zeit, als die zähringische Verwandtschaft sich ihrerseits tatsächlich die Herzogswürde zu sichern wusste. So darf man bei den Hermannen ein Wissen um ihre herzogliche Abkunft vermuten, auch wenn es ih-

10 Krieg, Familie (wie Anm. 9) S. 309, 311.

11 Ebd. S. 312-315, 322-331. - Ders.: Kaiser Sigismund, die Markgrafen von Baden und die Kurpfalz. In: Kaiser Sigismund (1368-1437). Zur Herrschaftspraxis eines europäischen Monarchen. Hg. von Karel Hruza und Alexandra Kaar (Forschungen zur Kaiser- und Papstgeschichte des Mittelalters, Beihefte zu J.F. Böhmer, Regesta Imperii 31). Wien/Köln/Weimar 2012. S. 175-196.

12 Siehe dazu Krieg, Adel (wie Anm.3) S.74-76 Anm.44. - Sönke Lorenz: Weilheim im frühen und hohen Mittelalter: Von der alemannischen Besiedlung bis zum Wegzug der Bertolde. In: Weilheim. Die Geschichte der Stadt an der Limburg. Hg. von Manfred Waßner. Kirchheim unter Teck 2007. S. 31-68. Hier S. 49, 52. - Thomas Zotz: Besigheim und die Herrschaftsentwicklung der Markgrafen von Baden. In: Das Land am mittleren Neckar zwischen Baden und Württemberg. Hg. von Hansmartin Schwarzmaier und Peter Rückert (Oberrheinische Studien 24). Ostfildern 2005. S. 73-94. Hier S.76-79. - Anders dagegen Eduard Hlawitschka: Zur Abstammung Richwaras, der Gemahlin Herzog Bertholds I. von Zähringen. In: ZGO 154 (2006) S. 1-20. 
nen im Unterschied zu den Zähringern nicht gelingen sollte, - sozusagen wieder zur Herzogswürde aufzusteigen. Letztlich bleibt das aber eine Vermutung.

Größere Sicherheit ist dagegen bei der Frage nach der Bedeutung der Markgrafenwürde zu gewinnen. Die Rangerhöhung Hermanns I. zum Markgrafen blieb, wie schon Karl Schmid betont hat, zweifellos konstitutiv für das Selbstverständnis der Hermanne und des Hauses Baden ${ }^{13}$. So treten die Hermanne schon in der Formierungsphase des Geschlechts während des 12. Jahrhunderts fast ausnahmslos ${ }^{14}$ mit dem Markgrafentitel in Erscheinung. Dabei findet man zunächst in der überwiegenden Zahl der Belege noch den bloßen Markgrafentitel ohne Zubenennung ${ }^{15}$, bevor dann allmählich etwa seit der Mitte des 12. Jahrhunderts die Zubenennung „von Baden“ üblich wird" ${ }^{16}$.

Das erste Zeugnis für die Zubenennung nach dem Ort Baden findet sich in der erwähnten Urkunde Kaiser Heinrichs V. aus dem Jahr 1112. Tatsächlich gewinnt der namengebende Sitz Baden aber erst mit einiger Verzögerung seit Mitte des 13. Jahrhunderts deutlichere Konturen als Herrschaftszentrum, nämlich in der Zeit des 1288 verstorbenen Markgrafen Rudolf I. ${ }^{17}$. Der Erstbeleg in der Herrscherurkunde vom 27. April 1112 steht in diesem Jahr im Übrigen keineswegs als singuläres Zeugnis da. Vielmehr firmiert Hermann II. bereits zweieinhalb Monate später, am 16. Juli 1112, erneut als Markgraf von Baden, und zwar wiederum unter den Intervenienten einer Urkunde Kaiser Heinrichs V. ${ }^{18}$. Die nächsten Bezeugungen dieser Zubenennung nach Baden (de Bathen bzw. de Badin) folgen dann erst wieder in einer Urkunde Lothars III. vom 6. Februar 1130 und zwei Tage später in einer Urkunde Bischof Ulrichs II. von Konstanz ${ }^{19}$.

13 Schmid, Werdegang (wie Anm. 8) S. 49.

${ }^{14}$ Zum Auftreten des comes-Titels vgl. etwa Regesten der Markgrafen von Baden und Hachberg 1050-1515. Bd. 1: Markgrafen von Baden 1050-1431, Markgrafen von Hachberg 1218-1428. Hg. von Richard Fester. Innsbruck 1900 (künftig: RMB) Nr. 11, 13, 29, 33, 35. MGH DD H IV, Nr. 126, 474.

15 Vgl. RMB 1 (wie Anm. 14) Nr. 14, 17-27, 31 f., 34, 36-51, 55-57, 97, 125, 128, 141. - MGH DD H IV, Nr. 281 (F), 468. - MGH DD K III, Nr. 22, 25, 35, 40, 42, 44, 52 f., 56 f., 59, 89 f., 92 , 246. - MGH DD F I, Nr. 101, 149, 573.

${ }^{16}$ Eine Durchsicht der entsprechenden Belege ergibt, dass sich diese Form der Benennung erst in Urkunden König Konrads III. und zwar etwa seit dem Jahr 1145 durchsetzt. Siehe RMB 1 (wie Anm. 14), Nr. 28, 30, 52f., 58, 77 f., 79a, 84, 130f., 140, 142-146, 146a (F), 148152, 154-156 (von hier an liefern die Markgrafenregesten keine besondere Kennzeichnung mehr, wenn der Titel „Markgraf von Baden“ lautet). - MGH DD K III, Nr. 18, 20, $36 \mathrm{f} .$, 72, 130, 182, 198-202, 204, 221, 235, 245, 267-269, 273, 289 (F). - MGH DD F I, Nr. 36, 52 f., 65, 69, 74, 97 f., 103, 110, 112, 123, 128, 133, 206f., 228, 310, 374, 779, 847, 1032 (F), 1042 (F).

17 Krieg, Herrschaftsbildung (wie Anm. 9) S. 166-168. - Kurt Andermann: Baden-Baden. In: Fürstliche Höfe und Residenzen im spätmittelalterlichen Reich. Ein dynastisch-topographisches Handbuch. Hg. von Werner Paravicini, Jan Hirschbiegel und Jörg Wettlaufer. Ostfildern 2003. Bd.2. S. 28-30. - Ders.: Baden-Badens Weg zur Residenz. In: ZGO 144 (1996) S. 259-269.

18 RMB 1 (wie Anm. 14) Nr. 30.

19 Ebd. Nr. 52 f. 
Dass die Zubenennung nach Baden aufkam, hängt sicherlich mit der alten Tradition des Ortes zusammen. Baden war nicht nur altes Königs- oder Reichsgut, sondern die warmen Quellen führten schon in der Antike zu einer Besiedelung dieses Ortes $^{20}$. Die baulichen Überreste aus der Antike, die hier im Mittelalter noch an das altrömische Kaisertum erinnerten, verliehen diesem Ort in den Augen der Zeitgenossen zweifellos höchstes Prestige.

Dennoch blieb für die Markgrafen von Baden im 12. und 13. Jahrhundert weiterhin der Bezug auf die Veroneser Markgrafenwürde von entscheidender Bedeutung. Denn nach der Zeit Markgraf Hermanns I. von Verona wurden dessen Nachfolger seit Markgraf Hermann III. immer wieder auch als Markgrafen „von Verona“ tituliert ${ }^{21}$. Diese Zubenennung nach Verona wird insbesondere im Zusammenhang mit der staufischen Italienpolitik fassbar. Auf den Italienzügen der Staufer agierten die Markgrafen als treue Parteigänger des Königtums, tatsächlich waren sie aber nicht dazu in der Lage, ihren Herrschaftsanspruch in der Mark Verona auf Dauer zur Geltung zu bringen. Sie hielten aber an der Selbstbezeichnung als Markgrafen von Verona bis zum Ende des 13. Jahrhunderts fest. Von wesentlicher Bedeutung sind in diesem Zusammenhang die Siegel der frühen Markgrafen von Baden: Dort prangte nämlich in der Umschrift noch Ende des 13. Jahrhunderts unter Markgraf Rudolf I. die stolze Selbstbezeichnung marchio de Verona ${ }^{22}$. Die Markgrafen von Baden, wie wir sie ganz selbstverständlich nennen, bezeichneten sich also auf Ihren Siegeln, die ja sicher zu den wichtigsten Zeugnisse ihrer herrscherlichen Selbstdarstellung gehörten, auffälligerweise gerade nicht nach Baden! Erst nach der Zeit des 1288 verstorbenen Markgrafen Rudolf I. bricht diese Verona-Tradition ab, die für

20 Siehe dazu Helmut Maurer: Baden-Baden. In: Die deutschen Königspfalzen. Repertorium der Pfalzen, Königshöfe und übrigen Aufenthaltsorte der Könige im deutschen Reich des Mittelalters. Bd. 3: Baden-Württemberg. 1. Lieferung. Göttingen 1988. S. 8-17. - Hansmartin Schwarzmaier: Baden-Baden im frühen Mittelalter. Die schriftliche Überlieferung aus den Klöstern Weißenburg und Selz. Baden-Baden 1988. - Erik Beck: Von Tempeln, Thermen und Toponymen - Zur Wahrnehmung und Funktion römischer Überreste am Oberrhein im Mittelalter. In: Antike im Mittelalter. Fortleben, Nachwirken, Wahrnehmung. 25 Jahre Forschungsverbund Archäologie und Geschichte des ersten Jahrtausends in Südwestdeutschland. Hg. von Heiko Steuer u. a. (Archäologie und Geschichte, Freiburger Forschungen zum ersten Jahrtausend in Südwestdeutschland 19) (im Druck).

21 Siehe dazu RMB 1 (wie Anm. 14) Nr. 80, 93 (= MGH DD K III, Nr. 255), 126, 133, 133a, 135, 139, 147. - MGH DD F I, Nr. 94, 96, 99, 107, 109, 111 (verunechtet), 114-116, 129, 218, 278, 315, 848f. - Christoph Friedrich Stälin: Wirtembergische Geschichte. Bd. 2. Stuttgart 1847. S. 338, 349. Vgl. dazu und auch zum Folgenden Krieg, Baden (wie Anm. 1) S. 37-40. Schmid, Werdegang (wie Anm. 8) S.66f. - Hansmartin Schwarzmaier: Die Markgrafen von Baden und Verona. In: König - Kirche - Adel. Herrschaftsstrukturen im mittleren Alpenraum und angrenzenden Gebieten (6.-13. Jahrhundert). Hg. von Rainer Loose und Sönke Lorenz. Lana 1999. S. 229-247.

${ }^{22}$ Dieser Titel erscheint sowohl auf den ersten überlieferten Markgrafensiegeln, die aus der Zeit Markgraf Hermanns V. stammen, als auch auf den Siegeln Markgraf Rudolfs I. aus den Jahren 1253 und 1286. Siehe dazu und auch zum Folgenden (mit weiterer Literatur) Krieg, Herrschaftsbildung (wie Anm. 9) S. 170 f. - Ders., Baden (wie Anm. 1) S. 39. 
das Selbstverständnis der Markgrafen anscheinend so zentral war, dass man auch noch einige Zeit nach dem realen Verlust der Mark Verona den Anspruch auf den daran geknüpften Markgrafentitel nicht aufgeben wollte. Interessanterweise taucht diese Veronatradition zu Anfang des 15. Jahrhunderts im Rahmen einer sagenhaften Herkunftserzählung plötzlich wieder auf ${ }^{23}$.

\section{Die Markgrafen im Gebiet von Neckar und Murr}

Bei den frühen Markgrafen spielte aber auch noch ein anderer räumlicher $\mathrm{Be}-$ zugspunkt eine Rolle, der sich im mittleren Neckargebiet befand. Denn in einer auf den 28. Februar 1100 datierten Urkunde des Klosters Schaffhausen ist Markgraf Hermann II. unmittelbar nach Herzog Bertold II. von Zähringen als marchio de Lintbburch, also als Markgraf von Limburg ${ }^{24}$, bezeugt. Daneben erscheint Hermann II. noch ein weiteres Mal als marchio de Limpurch in einem Zwiefaltener Nekrolog, wobei der Zusatz de Limpurch in diesem Fall aber erst in der Abschrift eines Zwiefaltener Mönchs von 1647 überliefert ist ${ }^{25}$. Das im zweiten Jahrzehnt des 12. Jahrhunderts entstandene Zwiefaltener Nekrolog liefert dann einen weiteren Beleg, der Hermanns II. gleichnamigen Vater betrifft. Markgraf Hermann I. wird dort nämlich ebenfalls der Limburg zugeordnet, jedoch nicht wie sein Sohn als „Markgraf von Limburg“, sondern als „Graf [von] Limburg“, wobei er auch als Mönch gekennzeichnet ist ${ }^{26}$. Die Zubenennungen nach Limburg, welche die frühen Markgrafen zunächst, wenn auch nur in wenigen Zeugnissen, eher als Markgrafen von Limburg denn als Markgrafen von Baden erscheinen lassen, verdienen

${ }^{23}$ Krieg, Baden (wie Anm. 1) S. 37 f., 40. - Ders., Familie (wie Anm. 9) S. 313-315.

${ }^{24}$ Parlow (wie Anm. 4) Nr. 154 S. 109. Zur Datierung der Niederschrift der Urkunde siehe Katalog der Urkunden und verwandten Aufzeichnungen von Schaffhausen bis 1150. In: Das Kloster Allerheiligen in Schaffhausen. Hg. von Kurt Bänteli, Rudolf Gamper und Peter Lehmann (Schaffhauser Archäologie. Monographien der Kantonsarchäologie Schaffhausen 4). Schaffhausen 1999. S.288-295. Hier S.291.

${ }^{25}$ MGH Necrologia Germaniae 1. Hg. von Franz Ludwig Baumann. Berlin 1888 (= MGH Necr. 1). S. 262 (7. Oktober). Siehe dazu Schmid, Baden-Baden (wie Anm. 1) S. 11 Anm. 54. - Rolf Kuithan, Die Benediktinerabtei Zwiefalten in der kirchlichen Welt des 12. Jahrhunderts. Ein Beitrag zur Untersuchung der Zwiefalter Memorialquellen (Bestandteil des Quellenwerkes Societas et Fraternitas). Phil. Diss. Münster 1997. Hier bes. S. 43-47.

${ }^{26}$ MGH Necr. 1 (wie Anm. 25). S. 250 (26. April): Heriman m., com. [de] Lintburk. Löst man die Abkürzungen auf, so ergibt sich Heriman monachus, comes [de] Lintburk, wobei das de eine sinngemäße Ergänzung des Editors darstellt. Zur Datierung dieses Eintrags siehe Schmid, Werdegang (wie Anm. 8) S. 50 Anm. 23. - Kuithan (wie Anm. 25) S.19f. Ebd. S. 20 datiert die Anlage des Nekrologs zwischen dem Terminus post quem „sicher nach 1114, wahrscheinlich erst nach 1119“ und dem Terminus ante quem „vermutlich schon im Frühjahr 1120, sicher 1122“. Der Eintrag zu Hermann I. gehört allem Anschein nach noch in diese Zeit. In einer - späteren - Überarbeitung des Nekrologs wurde im Übrigen Lintburk durch Leinziburc ersetzt. MGH Necr. 1 (wie Anm. 25). S. 250 Anm. h. 
es, ernst genommen zu werden. Denn sie deuten darauf hin, dass die Limburg zumindest aus der Sicht Schaffhausener und Zwiefaltener Mönche, und zwar auch noch nach dem Erstbeleg der Zubenennung nach Baden im Jahr 1112, für Hermann II. sicher, aber vielleicht auch schon für Hermann I. als Herrschaftszentrum von herausragender Bedeutung war, bevor sich schließlich die Burg Baden als zentraler Sitz etablierte ${ }^{27}$. Offensichtlich steht am Anfang der Formierung der Hermanne als Geschlecht, als sich dieses gegenüber dem zähringischen Zweig der Bertolde als eigene und davon klar unterschiedene Adelsfamilie herauszubilden begann, mit der Burg Limburg zunächst der alte Sitz der Bertolde beziehungsweise Herzog Bertolds I.

Die Limburg im mittleren Neckarraum scheint somit am Anfang des 12. Jahrhunderts, wenn auch nur schlaglichtartig, als räumlicher Bezugspunkt der Hermanne auf, der dadurch schon deutlich die Absonderung dieses sozusagen „hermannischen Zweigs“ der älteren Bertolde gegenüber dem Herzogsgeschlecht der Zähringer markiert. So ist es wohl mehr als nur Zufall, dass die erwähnte Schaffhausener Urkunde des Jahres 1100, in der Hermann II. als Markgraf von Limburg fassbar ist, zugleich den urkundlichen Erstbeleg für die Zubenennung Herzog Bertolds II. nach der Burg Zähringen bietet.

Vor diesem Hintergrund erklärt es sich, warum die Limburg, die irgendwann vor der Mitte des 12. Jahrhunderts in die Hand der Zähringer übergegangen zu sein scheint $^{28}$, auf der vorliegenden Karte (Abb. 2) noch als Besitz der Markgrafen von

27 Aufgrund der Zubenennungen nach der Limburg kann man Schmid, Baden-Baden (wie Anm. 8) S. 11 zufolge davon ausgehen, „daß die väterliche Burg bei Weilheim unter Teck der Sitz der beiden Hermanne gewesen ist." Ob das tatsächlich auch schon für Hermann I. gelten darf, bezweifelt Zotz, Besigheim (wie Anm.12) S. 83. - Zustimmend Florian Lamke: Die frühen Markgrafen von Baden, die Hessonen und die Zähringer. Konstellationen südwestdeutscher Adelsfamilien in der Zeit des Investiturstreits. In: ZGO 154 (2006) S. 21-42. Hier S. 41. - Zotz, Besigheim (wie Anm. 12) S. 83 macht zurecht darauf aufmerksam, dass es sich bei der Zuordnung Hermanns I. zur Limburg im Zwiefaltener Nekrolog auch um eine bloße Rückprojektion handeln könnte. Diese spiegelte dann aber zumindest die Situation beziehungsweise Wahrnehmung zur Zeit der Niederschrift des Nekrologs wider, so dass die herausragende Rolle, welche die Limburg für die Hermanne spielte, damit auch noch nach den ersten Belegen für die Zubenennung nach Baden im Jahr 1112 bezeugt ist.

${ }^{28}$ Zotz, Besigheim (wie Anm.12) S. 83 f. - Auf der Limburg saß damals eine offenbar von den Zähringern abhängige Familie. Siehe dazu künftig die Dissertation von Petra Skoda zum Thema „Herrschaft durch Gefolgschaft. Zur Bedeutung von Ministerialen, Freien und Adligen für die Herzöge von Zähringen im Einflußbereich ihres Hausklosters St. Peter“. - Siehe zur Limburg auch Christoph Bizer: Burgen der Kirchheimer Alb. In: Die Thietpoldispurch und die Burgen der Kirchheimer Alb. Neue Methoden und Ergebnisse der Burgenforschung. Hg. von dems. und Rolf Götz (Schriftenreihe des Stadtarchivs Kirchheim unter Teck 31). Kirchheim unter Teck 2004. S. 59-163. Hier S. 68-72. - Harald von der Osten-Woldenburg: Geophysikalische Prospektion auf der Limburg bei Weilheim an der Teck. In: Archäologische Ausgrabungen in Baden-Württemberg 2011. Stuttgart 2012. S. 294-297. - Anke K. Scholz: Die Stammburg der Zähringer - neue archäologische Ausgrabungen auf der Limburg bei Weilheim an der Teck. In: Archäologische Ausgrabungen in Baden-Württemberg 2011. 


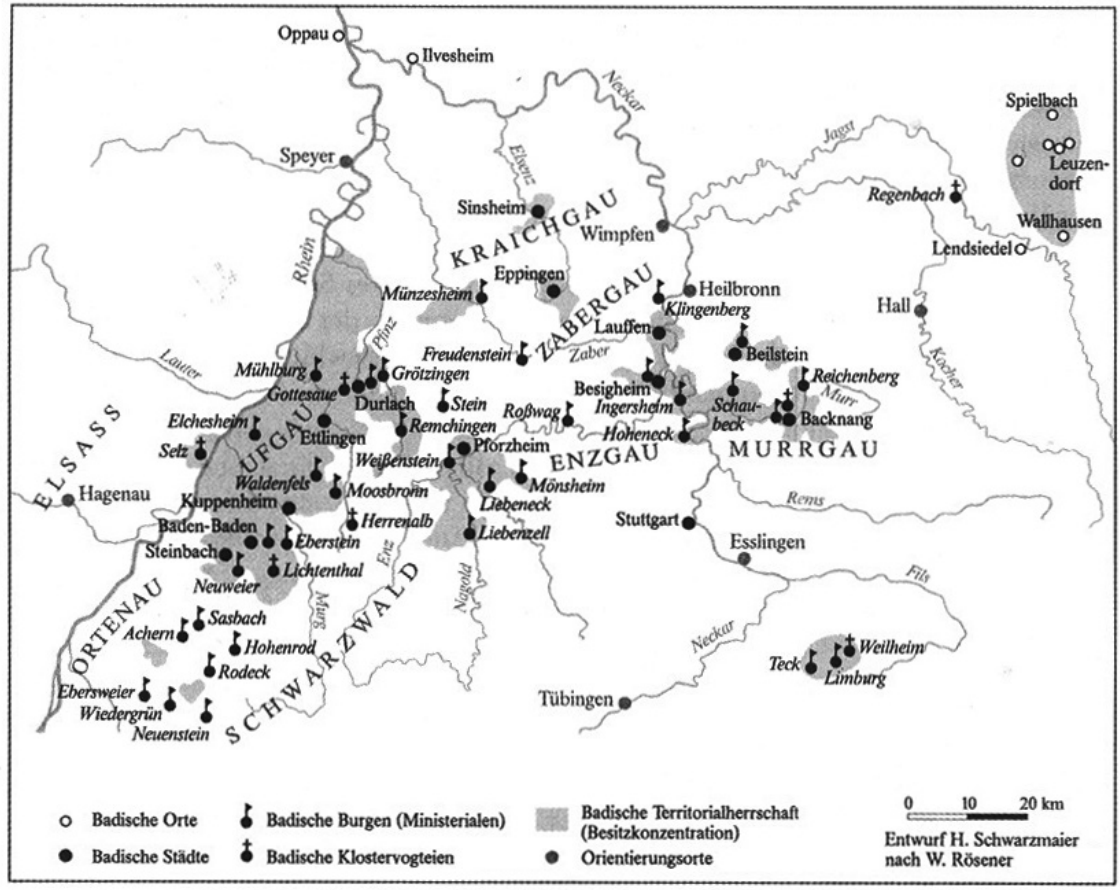

Herrschaftsgebiete der Markgrafen von Baden um 1250

Baden gekennzeichnet ist. Anders verhält es sich jedoch mit der Burg Teck und der Vogtei über das Kloster Weilheim, die abweichend von den Einträgen im Kartenbild tatsächlich nicht in markgräflicher Hand bezeugt sind. Vielmehr verfügte 1152 nachweislich Herzog Bertold IV. von Zähringen über die Burg Teck, wobei die Burg in diesem Zusammenhang überhaupt erstmals schriftlich belegt ist ${ }^{29}$. Die Burg Teck sollte dann nach dem Tod Herzog Bertolds IV. von Zähringen im Jahr

Stuttgart 2012. S. 297-301. Die Bezeichnung der Limburg als „Stammburg der Zähringer“ ist dabei aber streng genommen unzutreffend (siehe oben bei Anm. 3).

29 Parlow (wie Anm. 4) Nr.343 S. 225-227. Die möglicherweise von Herzog Konrad von Zähringen oder vielleicht auch schon vor 1100 von den Grafen von Nellenburg errichtete Burg Teck gelangte zusammen mit der zugehörigen Herrschaft wohl nach dem Tod des Grafen Burkhard von Nellenburg (wohl 21. Januar 1102) an Herzog Bertold II. von Zähringen. Siehe dazu Parlow Nr.156 S.110f. - Lorenz, Weilheim (wie Anm. 12) S. 62. - Zu Weilheim siehe ebd. S. 46-68. - Sönke Lorenz: Zur Geschichte des „verlegten“ Klosters Weilheim vor und nach 1093. In: Das Kloster St. Peter auf dem Schwarzwald. Studien zu seiner Geschichte von der Gründung im 11. Jahrhundert bis zur frühen Neuzeit. Hg. von Hans-Otto Mühleisen, Hugo Ott und Thomas Zotz (Veröffentlichung des Alemannischen Instituts Freiburg i. Br. 68). Waldkirch 2001. S. 11-32. 
1186 auch zum namengebenden Sitz einer Seitenlinie der Zähringer werden, nämlich der Herzöge von Teck ${ }^{30}$. Demgegenüber deuten jedoch die frühen Zubenennungen nach der Limburg darauf hin, dass dieser Burg zumindest vorübergehend als namengebendem Bezugspunkt und als frühem Herrschaftszentrum zu Beginn der Geschichte der markgräflichen Familie noch eine nicht zu vernachlässigende Bedeutung zukam.

Etwas später fassbar als dieser offenbar noch während des 12. Jahrhunderts an die Herzöge von Zähringen übergegangene Besitzkomplex um Limburg ist der markgräfliche Besitzschwerpunkt um Backnang, das für die Markgrafen von Baden bis zur Mitte des 13. Jahrhunderts zentrale Bedeutung behielt. Backnang fiel wohl durch die Ehe Hermanns II. von Baden mit Judith von Backnang in die Hand der Markgrafen von Baden ${ }^{31}$, wobei diese Heiratsverbindung an sich schon das besondere Interesse der frühen Markgrafen an diesem für sie zentralen Raum unterstreicht. Markgraf Hermann II. und seine Gemahlin Judith gründeten in Backnang, das wahrscheinlich als Erbe Judiths an die Markgrafen fiel, um 1116 das Augustinerchorherrenstift St. Pankratius, dessen Einrichtung dann 1122 auch vom Speyerer Bischof bestätigt wurde ${ }^{32}$. Das Pankratiusstift in Backnang fungierte bis zur Mitte des 13. Jahrhunderts als Grablege der Familie, bis schließlich 1248 die Umbettung Markgraf Hermanns V. von Backnang in das Zisterzienserinnenkloster Lichtenthal bei Baden die Verlagerung des Herrschaftsschwerpunktes an den Oberrhein und den damit einhergehenden Rückzug aus dem mittleren Neckarraum markierte ${ }^{33}$. Backnang war demzufolge das ältere, erste geistliche Zentrum der Herrschaft der Markgrafen von Baden. Für Backnang, das an einem für die Markgrafen wichtigen Verkehrsweg lag $^{34}$, wird dabei ein Ausbau zur Stadt noch

30 Siehe zur Burg Teck und zur Tecker Seitenlinie der Zähringer Parlow (wie Anm. 4) Nr. 232 S. 157, Nr. 523 S. 335 f. - Rolf Götz: Von der ersten urkundlichen Nennung im Jahre 960 bis zur Mitte des 16. Jahrhunderts. In: Kirchheim unter Teck. Marktort, Amtsstadt, Mittelzentrum. Hg. von Rainer Kilian. Kirchheim unter Teck 2006. S. 97-273. Hier S.116143. - Ders.: Die Herzöge von Teck. Herzöge ohne Herzogtum (Schriftenreihe des Stadtarchivs Kirchheim unter Teck 33). Kirchheim unter Teck 2009. - Bizer (wie Anm. 28) S. 72-82.

${ }^{31}$ Hierbei stütze ich mich auf Lamke, Markgrafen (wie Anm. 27) S. 26-33. Danach ist davon auszugehen, dass Hermanns II. Gemahlin die Hessonin Judith von Backnang war. Zustimmend Lorenz, Weilheim (wie Anm. 12) S. 63. - Zotz, Besigheim (wie Anm. 12) S. 85.

32 Siehe dazu WUB 1 Nr. 271 S. 343, Nr.276 S.348f., Nr. 282 S. 358f., und (mit weiterer Literatur) Lamke, Markgrafen (wie Anm. 27) S. 25 f., 42. - Zum Backnanger Nekrolog siehe Gerhard Fritz: Der Backnanger Nekrolog. Studien zur Geschichte des Augustiner-Chorherrenstifts Backnang. In: ZWLG 44 (1985) S. 11-64.

${ }^{33}$ RMB 1 (wie Anm. 14) Nr. 384, 386f., 390 f., 395 f., 447. - Krieg, Herrschaftsbildung (wie Anm. 9) S. 166-168. - Hansmartin Schwarzmaier: Lichtenthal als Grabkloster der Markgrafen von Baden im 13. und 14. Jahrhundert. In: 750 Jahre Zisterzienserinnen-Abtei Lichtenthal. Faszination eines Klosters. Hg. von Harald Siebenmorgen u. a. Sigmaringen 1995. S. 2334. - Pia Schindele: Die Abtei Lichtenthal. Ihr Verhältnis zum Cistercienserorden, zu Päpsten und Bischöfen und zum badischen Landesherrn im Laufe der Jahrhunderte. In: Freiburger Diözesanarchiv 104 (1984) S. 19-166. Hier S. 25-30.

${ }^{34}$ Rüdiger Stenzel: Die Städte der Markgrafen von Baden. In: Landesherrliche Städte in 
unter Markgraf Hermann V. angenommen. So ist etwa für 1231 ein Schultheiß erwähnt ${ }^{35}$, und um 1235 wurde Backnang zum Ziel der Anhänger König Heinrichs (VII.), die im Kampf gegen Markgraf Hermann V. das Kloster niederbrannten ${ }^{36}$. 1245 wird Backnang dann in einer Papsturkunde als oppidum bezeichnet, so dass man mit Haselier vermuten kann, dass die Stadterhebung wohl im Zuge des Wiederaufbaus Backnangs nach der Zerstörung durch die Anhänger König Heinrichs (VII.) erfolgt sein könnte ${ }^{37}$. Richter als städtische Amtsträger sind jedoch erst im Jahre 1297 bezeugt $^{38}$. Schon 1304 an die Grafen von Württemberg verpfändet, wurde die Stadt, deren erstes Siegel aus dem Jahr 1312 überliefert ist, 1324 endgültig württembergisch ${ }^{39}$.

Mit dem Stift Backnang steht auch die markgräfliche Burg Reichenberg in $\mathrm{Zu}$ sammenhang, die Markgraf Hermann V. offensichtlich zunächst eigenmächtig auf Grund und Boden des Stifts errichtet hatte. Diese widerrechtliche Handlung ließ er sich dann nachträglich im Jahre 1231 von den Stiftsklerikern legalisieren, indem er diesen dafür eine Entschädigung gab ${ }^{40}$. Aufgrund der besonders aufwändigen Gestaltung dieser Burganlage hat Hans-Martin Maurer die Auffassung vertreten, dass die Burg Reichenberg „ihrer Bestimmung nach mehr war als irgendein Adelssitz, nämlich ein Hauptsitz des Markgrafen selbst“, der möglicherweise sogar den älteren Sitz in Backnang ablösen sollte ${ }^{41}$. Ebenso wie Backnang war auch die Burg Reichenberg schon 1304 an Württemberg verpfändet und fiel damit ebenso an die Grafen von Württemberg ${ }^{42}$.

Südwestdeutschland. Hg. von Jürgen Treffeisen und Kurt Andermann (Oberrheinische Studien 12). Sigmaringen 1994, S. 89-130. Hier S. 96.

35 RMB 1 (wie Anm. 14) Nr. 298.

36 Siehe RMB 1 (wie Anm. 14) Nr. 298. - WUB 4 Nachtrag Nr. 123 S. 418-421. - Günter Haselier: Die Markgrafen von Baden und ihre Städte. In: ZGO 107 (1959) S. 263-290. Hier S. 269.

37 WUB 4 Nr. 1040 S. 90-94. - Haselier (wie Anm. 36) S. 269.

38 WUB 11 Nr. 5035 S. 67. - Haselier (wie oben).

39 RMB 1 (wie Anm. 14) Nr. 665. - Haselier (wie Anm. 36) S. 269f. - Württembergisches Städtebuch. Hg. von Erich Keyser (Deutsches Städtebuch. Handbuch städtischer Geschichte IV. 2). Stuttgart 1962. S. 38 (10 a, 12). Vgl. zum Vordringen Württembergs Gerhard Fritz: Die Markgrafen von Baden und der mittlere Neckarraum. In: ZWLG 50 (1991) S. 51-66. Hier S. 64-66.

40 WUB 3 Nr. 783 S. 276f. - RMB 1 (wie Anm. 14) Nr. 298.

${ }^{41}$ Hans-Martin Maurer: Die Türme des Markgrafen Hermann V. im Rahmen stauferzeitlicher Wehrbau-Architektur. In: Das Land am mittleren Neckar zwischen Baden und Württemberg. Hg. von Hansmartin Schwarzmaier und Peter Rückert (Oberrheinische Studien 24). Ostfildern 2005. S.111-144. Hier S. 140: „Wenige Kilometer nördlich von Backnang gelegen, sollte sie als Höhenburg wohl die alte dortige Residenz ablösen." Nach den Erwerbungen Hermanns V. im Jahr 1219 habe sich dieser dann aber weiter nach Westen orientiert und wohl den Plan gefasst, sich in Besigheim eine neue Residenz zu schaffen. Ebd. Siehe dazu unten bei Anm. $66 \mathrm{f}$.

${ }^{42}$ RMB 1 (wie Anm. 14) Nr. 665. 
Die an der Wegverbindung von Backnang nach Klingenberg beziehungsweise Lauffen gelegene Stadt Beilstein ist 1304 erstmals ausdrücklich als solche bezeugt $^{43}$. Es handelt sich dabei um eine typische Burgsiedlung, wobei die Burg zunächst 1230/1231 im Besitz eines Grafen Bertold von Beilstein fassbar ist ${ }^{44}$. Dieser Graf Bertold tätigte 1230 eine Schenkung an das Stift Backnang, weshalb bei ihm eine Verbindung zu den Backnanger Hessonen vermutet wird ${ }^{45}$. Danach gelangte Beilstein an die Markgrafen von Baden, wobei 1290 Markgraf Hermann IV. sicher als Lehnherr der Burg bezeugt ist: Damals verlieh er seinem Diener Friedrich von Gomaringen ein Burglehen, das diesen zu Diensten in Backnang oder Beilstein verpflichtete $^{46} .1294$ ist ein Ritter Dietrich Kieme von Beilstein als Diener der Markgräfin Agnes von Baden fassbar ${ }^{47} .1304$ ließ sich dann der Württemberger Graf Eberhard der Erlauchte von König Albrecht den ungestörten Besitz von Burg und Stadt Beilstein zusammen mit der Burg Reichenberg und der Stadt Backnang bestätigen $^{48}$. Es ist anzunehmen, dass dieser markgräfliche Besitz über die Ehe Eberhards des Erlauchten mit der Markgräfin Irmgard von Baden, der Tochter Markgraf Rudolfs I., an Württemberg gelangte ${ }^{49}$. Ob die Gründung der Stadt Beilstein bereits unter Markgraf Hermann V. von Baden oder doch erst unter seinem Sohn Rudolf I. erfolgte, lässt sich dabei nicht sicher entscheiden ${ }^{50}$.

Die wohl um die Wende vom 11. zum 12. Jahrhundert errichtete Burg Lauffen, nach der sich die Grafen von Lauffen benannten, gelangte nach dem Aussterben der Familie an König Friedrich II. ${ }^{51}$. Lauffen gehörte zu denjenigen Stauferstädten, die Friedrich II. dann im Rahmen der Regelung der pfälzischen Erbschaft 1219 an Hermann V. von Baden übertrug, und zwar als Gegenleistung dafür, dass der

43 Ebd.

44 WUB 4 Nr.112 S.409f. - Fritz, Markgrafen (wie Anm. 39) S. 59. - Hermann Ebmer: Beilstein. In: Der Landkreis Heilbronn 1 (Baden-Württemberg - Das Land in seinen Kreisen). Ostfildern 2010. S. 354-358. Hier S. 354-356. - Stenzel (wie Anm. 34) S. 98.

45 Fritz, Markgrafen (wie Anm. 39) S. 59 f.

46 RMB 1 (wie Anm. 14) Nr. 588. - Fritz, Markgrafen (wie Anm. 39) S. 60.

47 RMB 1 (wie Anm. 14) Nr. 616. - Siehe dazu Rösener (wie Anm. 9) S. 57. - 1284 erscheinen ein Dietrich von Bilstein und 1285 ein Harmann genannt Kriech von Beilstein, die Ehmer (wie Anm. 44) S. 354 zufolge ebenfalls Dienstleute der Markgrafen von Baden gewesen sein könnten.

${ }^{48}$ RMB 1 (wie Anm. 14) Nr. 665. Siehe dazu oben bei Anm. 39, $42 \mathrm{f}$.

49 Fritz, Markgrafen (wie Anm. 39) S. 63 f.

$50 \mathrm{Zu}$ Hermann V. als möglichem Stadtgründer Fritz, Markgrafen (wie Anm. 39) S. 63 f. Stenzel (wie Anm. 34) S. 98. - Dagegen sieht Ehmer (wie Anm. 44) S. 355 Rudolf I. als Stadtgründer an.

51 Sönke Lorenz: Lauffen. In: LexMA 5. München/Zürich 1991. Sp.1756. - Ders.: Im Dunkel des frühen Mittelalters: Zur Geschichte Besigheims - Rahmenbedingungen und Strukturen. In: Das Land am mittleren Neckar zwischen Baden und Württemberg. Hg. von Hansmartin Schwarzmaier und Peter Rückert (Oberrheinische Studien 24). Ostfildern 2005. S. 11-72. Hier S. 69-71. - Norbert Hofmann: Vielfalt der alten Ordnung (bis 1806). In: Der Landkreis Heilbronn 2 (Baden-Württemberg - Das Land in seinen Kreisen). Ostfildern 2010. S. 90-96. Hier S. 90. 
Markgraf auf die Braunschweigischen Erbgüter seiner Gemahlin Irmgard verzichtete. Aus der Hand des Königs erhielt Markgraf Hermann damals die Stadt Ettlingen als Lehen, Durlach als Eigenbesitz und darüber hinaus die Städte Lauffen, Sinsheim und Eppingen als Pfandschaften ${ }^{52}$. Wie zentral diese Erwerbungen für den Markgrafen von Baden waren, spiegelt die älteste erhaltene Königsurkunde des markgräflich-badischen Archivs wider, die Friedrich II. 1234 für Hermann V. ausstellen und mit einer Goldbulle besiegeln ließ ${ }^{53}$. Nachdem in Lauffen bereits 1231 Vogt und Schultheiß bezeugt sind, bietet die Goldbulle Friedrichs II. aus dem Jahr 1234 auch die erste Erwähnung der civitas Lauffen ${ }^{54}$. Nach dem ältesten Siegel der Bürger von Lauffen aus dem Jahr 1299 findet sich von 1311 bis 1343 der badische Schild als Siegelbild ${ }^{55}$. Auch Lauffen fiel schließlich im Lauf des 14. Jahrhunderts an Württemberg. Burg und Stadt wurden von den Markgrafen von Baden zunächst an die Hofwart von Kirchheim 1343 verpfändet und 1346 verkauft, bevor schließlich 1361 und 1369 Württemberg diesen Besitz erwarb ${ }^{56}$.

Demgegenüber erscheinen die nach der bei Heilbronn gelegenen Burg Klingenberg zubenannten Herren noch 1381 unter den markgräflich-badischen Lehnsleuten $^{57}$. Schon 1291 bezeugte ein Herr von Klingenberg die Belehnung Markgraf Hermanns VII. durch den Weißenburger $\mathrm{Abt}^{58}$. Und ein Reinbot von Klingenberg begegnet 1295 im Gefolge des Markgrafen Friedrich II. von Baden ${ }^{59}$. So ist Rösener zufolge wohl anzunehmen, dass die Markgrafen von Baden in Klingenberg ebenso wie auch anderenorts „schon seit der Mitte des 13. Jahrhunderts als Erben und Nachfolger der Staufer von der Abtei Weißenburg die Lehnshoheit über Burg und Dorf und das Patronatsrecht" erhalten haben ${ }^{60}$. Erstmals sicher belegt ist die Belehnung der Markgrafen von Baden durch den Abt von Weißenburg jedoch erst im Jahre $1339^{61}$.

Auf der Burg Schaubeck saß die badische Ministerialenfamilie der Schobelin beziehungsweise von Schaubeck, die „eine hervorragende Rolle in der Verwaltung und Sicherung des badischen Herrschaftsbereichs am mittleren Neckar" spielte ${ }^{62}$.

52 RMB 1 (wie Anm. 14), Nr. 227, Nr. 343. - Hansmartin Schwarzmaier: Baden. In: Handbuch der baden-württembergischen Geschichte. Bd. 2: Die Territorien im Alten Reich. Hg. von Meinrad Schaab und Hansmartin Schwarzmaier. Stuttgart 1995. S. 164-246. Hier S. $179 \mathrm{f}$. - Fritz, Markgrafen (wie Anm. 39) S. 58f. - Haselier (wie Anm. 36) S. 265.

53 Vgl. Schwarzmaier, Baden (wie Anm. 52) S. 180 zu RMB 1 (wie Anm. 14) Nr. 343.

54 Hofmann (wie Anm. 51) S. 91.

55 Ebd.

56 Ebd. S. 90. - Fritz, Markgrafen (wie Anm. 39) S. 65.

57 Bernhard Theil: Das älteste Lehnbuch der Markgrafen von Baden (1381). Edition und Untersuchung. Ein Beitrag zur Geschichte des Lehnswesens im Spätmittelalter (VKgL A 25). Stuttgart 1974. S. 120, 211.

58 RMB 1 (wie Anm. 14) Nr. 595. - Siehe auch zum Folgenden Rösener (wie Anm. 9) S. 56.

59 RMB 1 (wie Anm. 14) Nr. 627. Vgl. auch ebd. Nr. 616, Nr. 1291.

60 Rösener (wie Anm. 9) 56.

61 RMB 1 (wie Anm. 14) Nr. 977.

62 Rösener(wie Anm. 9) S. 55. 
Während des 13. Jahrhunderts fungierten mehrere Familienmitglieder als markgräfliche Vögte in Besigheim, wobei sich namentlich Konrad Marschall von Besigheim, ein Bruder Bertolds von Schaubeck, von 1285 bis 1296 häufig im markgräflichen Gefolge aufhielt ${ }^{63}$.

Der Hof Besigheim gelangte im Jahr 1153 an Markgraf Hermann III. von Baden, und zwar als Geschenk König Friedrichs I. Barbarossa, der erst im Jahr zuvor den Thron erlangt hatte. Diese Schenkung ist, worauf Thomas Zotz und Stefan Weinfurter hingewiesen haben, zweifellos als deutliches Zeichen der Nähe der Markgrafen von Baden zu den Staufern zu werten ${ }^{64}$. Offensichtlich ging es Friedrich Barbarossa bei dieser Besitzübertragung darum, den Markgrafen Hermann III. als einen seiner treuesten Anhänger mit dieser wichtigen, verkehrsgünstig gelegenen Position auszustatten. Das wird nicht zuletzt dadurch unterstrichen, dass dieser Schenkung erst noch die Äbtissin von Erstein zustimmen musste, denn das Stift Erstein hatte den ehemaligen Königshof Besigheim einst von der Kaiserin Agnes, der Gemahlin Kaiser Heinrichs III., als Geschenk erhalten. Doch für Friedrich Barbarossa war die Sicherung der Hilfe und Loyalität seines Gefolgsmannes Markgraf Hermann III. offensichtlich wichtiger als die Wahrung der überkommenen Rechte der Ersteiner Stiftsdamen ${ }^{65}$. Vor allem Markgraf Hermann V. von Baden wusste im Rahmen der Westerweiterung seiner Herrschaft im Raum zwischen Neckar und Oberrhein diesen Stützpunkt zu nutzen. Er ließ hier in äußerst anspruchsvoller Weise eine Residenz mit zwei Burgen und einer dazwischen gelegenen Stadt errichten, die „den hervorgehobenen Status des Bauherrn augenfällig“ machen sollte $e^{66}$. Davon zeugen bis heute die beiden mächtigen Türme, die immer noch das Stadtbild Besigheims prägen ${ }^{67}$. Die Stadt am Zusammenfluss von Enz und Neckar blieb im Übrigen noch bis ins 16. Jahrhundert hinein in den Händen der Markgrafen von Baden, bevor sie schließlich ebenfalls an Württemberg fiel ${ }^{68}$.

Seit der Zeit Markgraf Hermanns V. von Baden sind badische Ministeriale fassbar, die ihren Stammsitz auf der Burg Ingersheim hatten, die sie von den Markgrafen von Baden zu Lehen trugen ${ }^{69}$. Nach dem Aussterben dieser Familie um 1320,

${ }^{63}$ Ebd. S. 55 f.

${ }^{64}$ Siehe MGH DD F I, Nr. 65. Vgl. auch zum Folgenden Zotz, Besigheim (wie Anm. 12). - Stefan Weinfurter: Herrschaftsbildung in staufischer Zeit mit Blick auf den unteren Neckar. In: Das Land am mittleren Neckar zwischen Baden und Württemberg. Hg. von Hansmartin Schwarzmaier und Peter Rückert (Oberrheinische Studien 24). Ostfildern 2005. S. 95-109. - Hansmartin Schwarzmaier: Besigheim zwischen König und Markgraf. Zur Urkunde vom 12. Juli 1153 (Besigheimer Geschichtsblätter 23). Besigheim 2003.

${ }_{65}$ Zotz, Besigheim (wie Anm. 12) S. 73-75. - Schwarzmaier, Besigheim (wie Anm. 64).

66 Maurer, Türme (wie Anm. 41) S. 139.

${ }^{67} \mathrm{Zu}$ Burg und Stadt Besigheim siehe Maurer (wie Anm. 41) S.111-144. - Hansmartin Schwarzmaier: Von der Burg zur Stadt: Zur Stadtwerdung von Besigheim. In: Das Land am mittleren Neckar zwischen Baden und Württemberg. Hg. von dems. und Peter Rückert (Oberrheinische Studien 24). Ostfildern 2005. S. 145-162.

${ }^{68}$ Fritz, Markgrafen (wie Anm. 39) S. 66.

${ }^{69}$ Rösener (wie Anm. 9) S. 57 f. Hermann V. bestätigte 1242 die Schenkung seines Ministe- 
fiel das Erbe in Ingersheim an Heinrich Sturmfeder und blieb damit in der Hand einer weiteren markgräflichen Dienstmannenfamilie, die sich seit der zweiten Hälfte des 13. Jahrhunderts nach der markgräflichen Burg von Oppenweiler zubenannte ${ }^{70}$. Mit dem bereits wiederholt erwähnten Übergang von Backnang, Reichenberg und Beilstein an Württemberg fielen auch die Lehnsrechte über die Güter der Sturmfeder in Oppenweiler an die Grafen von Württemberg ${ }^{71}$. 1504 wurde schließlich auch Ingersheim württembergisch, nachdem Württemberg bereits 1342 „hier Fuß gefaßt" hatte ${ }^{72}$.

Eine weitere badische Position in diesem Raum war die Burg Hoheneck ${ }^{73}$. Die Hack von Hoheneck, von denen man nicht sicher sagen kann, ob sie ursprünglich markgräfliche Ministerialen waren, standen in markgräflich-badischem Lehnsverhältnis. 1291 bestätigt Markgraf Hermann VII. als Lehnsherr die Schenkung von Gütern an das Kloster Bebenhausen durch Albert Hack von Hoheneck und dessen Sohn Rudolf ${ }^{74} .1360$ erwarben auch hier die Grafen von Württemberg Rechte an Burg und Stadt. Der pfandweise erneut an die Hacken von Hoheneck ausgegebene Besitz wurde schließlich gegen Ende des 15. Jahrhunderts von Herzog Eberhard II. wieder ausgelöst und damit von Württemberg vollständig übernommen ${ }^{75}$.

Da Marbach in diesem Band von Sönke Lorenz behandelt wird, ist an dieser Stelle nicht näher darauf einzugehen. Im Übrigen fehlen für den Zeitraum zwischen der urkundlichen Bestätigung von Markt und Zoll in der villa Marbach, die sich Bischof Walther von Speyer im Jahre 1009 von König Heinrich II. ausstellen ließ, und der Erwähnung eines Marbacher Leutpriesters in einer Schiedsgerichtsurkunde des Jahres 1244 leider jegliche Schriftzeugnisse. Gesicherte Erkenntnisse darüber, wann genau und wie die Markgrafen von Baden hier zwischenzeitlich Herrschaftsrechte erlangen konnten, sind daher, wie der Beitrag von Sönke Lorenz aufzeigt, kaum möglich ${ }^{76}$.

Seit den Forschungen von Hansmartin Decker-Hauff wird die Gründung der Stadt Stuttgart einer Initiative der Markgrafen von Baden zugeschrieben, wobei als Stadtgründer Hermann V. gilt ${ }^{77}$. Stuttgart fiel bereits um 1245 wohl als Mitgift

rialen Friedrich von Ingersheim an das Kloster Denkendorf. Ebd. S. 57. - RMB 1 (wie Anm. 14) Nr. 380.

70 Rösener (wie Anm. 9) S. $57 \mathrm{f}$.

71 Ebd. S. 58.

72 OAB Besigheim. Stuttgart 1853. S. 192.

73 Rösener (wie Anm. 9) S. 83 f.

74 Ebd. - RMB 1 (wie Anm. 14) Nr. 593 f. - LB-W 3. Stuttgart 1978. S. 426 zufolge sei die bei der Burg angelegte Siedlung von den Hack von Hoheneck mit Zustimmung der Markgrafen von Baden zur Stadt erhoben worden und 1345 erstmals als solche belegt.

75 OAB Ludwigsburg. Stuttgart 1859. S. 239.

76 Siehe zu Marbach den Beitrag von Sönke Lorenz im vorliegenden Band.

77 Hansmartin Decker-Hauff: Geschichte der Stadt Stuttgart. Bd.1. Von der Frühzeit bis zur Reformation. Stuttgart 1966. S.129-155. - Stenzel (wie Anm. 34) S. 93-96. - Schwarzmaier, Baden (wie Anm. 52) S. 131. - Sönke Lorenz: Stuttgart. In: LexMA 8. München 1997. Sp.270f. - Oliver Auge: Stuttgart. In: Fürstliche Höfe und Residenzen im spätmittelalter- 
Mathildes, der Schwester der badischen Markgrafen Rudolf I. und Hermann VI., an die Grafen von Württemberg, genauer an deren Gemahl, den württembergischen Grafen Ulrich I., den Stifter ${ }^{78}$. Dieser Herrschaftswechsel von Baden zu Württemberg kann dabei, wie Sönke Lorenz in seinem nachfolgenden Beitrag betont, „nur relativ umständlich“ erschlossen werden ${ }^{79}$. Der Rückschluss auf diesen Wechsel der Stuttgarter Stadtherren basiert dabei nicht zuletzt auf der im Jahre 1259 ausgestellten Urkunde, mit der Graf Ulrich I. von Württemberg und seine Gemahlin Mathilde die in Stuttgart befindlichen Weinberge des Frauenklosters Pfullingen von sämtlichen Abgaben befreiten (Abb. 3).

Die Urkunde ist von Graf Ulrich von Württemberg und der Markgräfin Mathilde von Baden gemeinsam besiegelt, wobei auffällt, dass die Markgräfin Mathilde das Siegel ihrer Mutter Irmgard verwendete, da sie offensichtlich über kein eigenes verfügte ${ }^{80}$. Die dieser Abgabenbefreiung vorausgehende Schenkung der Weinberge an das Kloster wird in derselben Urkunde auch von Markgraf Rudolf I. von Baden bestätigt, dessen Reitersiegel in der Mitte zwischen den beiden bereits erwähnten Siegeln angebracht ist. Markgraf Rudolf gab hiermit ausdrücklich seine Zustimmung zur Schenkung der genannten Güter, als deren vorheriger Eigentümer er sich zugleich zu erkennen gibt. Diese Stelle findet sich am Ende des Urkundentextes im Anschluss an die Datierung in hellerer Tinte angefügt.

Die Urkunde bezeugt in jedem Fall ältere Besitzrechte der Markgrafen von Baden in Stuttgart, und auch darüber hinaus verweisen weitere Indizien auf ältere Herrschaftsrechte der Badener im räumlichen Umfeld der Stadt ${ }^{81}$. Davon, dass Stuttgart von den Markgrafen von Baden nicht nur als Herrschaftssitz ausgebaut, sondern auch bereits zur Stadt erhoben wurde, bevor es an die Grafen von Württemberg fiel, berichten jedoch erst späte historiographische Zeugnisse seit dem 16. Jahrhundert ${ }^{82}$. Sichere Belege für die Existenz einer städtischen Siedlung finden sich im Übrigen gegen Ende des 13. Jahrhunderts, als Stuttgart erstmals 1286 und 1290 als stat beziehungsweise civitas bezeugt ist ${ }^{83}$. Dennoch bleibt festzuhalten, dass die frühen Markgrafen von Baden im Raum Stuttgart offensichtlich bereits präsent waren, bevor die Grafen von Württemberg dort ihren zentralen Herr-

lichen Reich. Ein dynastisch-topographisches Handbuch. Hg. von Werner Paravicini, Jan Hirschbiegel und Jörg Wettlaufer. Ostfildern 2003. Bd. 2. S. 568-571. - Hansmartin Schwarzmaier: Der Ursprung Badens am mittleren Neckar. In: Baden! 900 Jahre (wie Anm. 2) S. 30.

78 Decker-Hauff (wie Anm. 77) S. 149. - Stenzel (wie Anm. 34) S. 95. - Lorenz, Stuttgart (wie Anm. 77) Sp. 270. - Auge (wie Anm. 77) S. 569.

79 Siehe Lorenz in diesem Band.

80 WUB 5 Nr. 1519 S. 286 f.

${ }^{81}$ Vgl. dazu auch Decker-Hauff (wie Anm. 77) S. 129-135. Den späteren Markgrafen von Baden könnte hier vielleicht über Richwara, die Gemahlin Bertolds I. von Kärnten, schwäbisches Herzogsgut zugekommen sein. Stenzel (wie Anm. 34) S. $94 \mathrm{f}$.

82 Siehe ebd. S. 135-138.

83 Auge (wie Anm. 77) S. 568. Siehe dazu ausführlicher den Beitrag von Peter Rückert in diesem Band. 


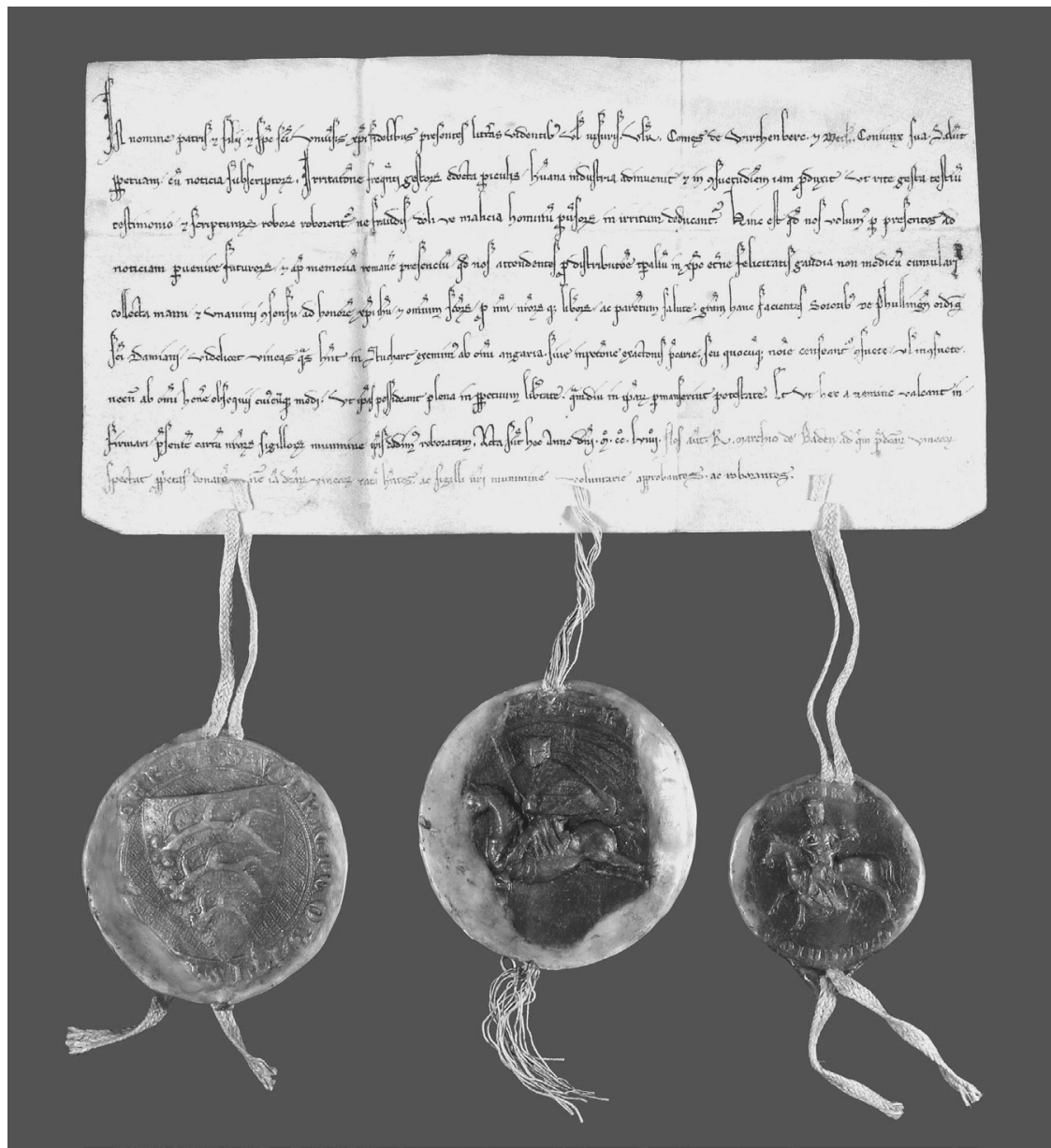

Abb. 3: Urkunde des Grafen Ulrich I. von Württemberg und seiner Gemahlin Mathilde von 1259 (Vorlage: HStA Stuttgart).

schaftssitz einrichteten und sich anschließend aus diesem Gebiet zurückzogen beziehungsweise verdrängt wurden.

Bereits um die Mitte des 13. Jahrhunderts und um 1300 verloren die Markgrafen durch zwei badisch-württembergische Heiraten ihre bisherige Herrschaftspositi- 
on im Neckar-Murr-Gebiet ${ }^{84}$. Die württembergischen Grafen übernahmen damit schon seit dem 13. Jahrhundert zentrale markgräfliche Besitzpositionen im mittleren Neckargebiet, die noch unter Hermann V. einen wichtigen Herrschaftsschwerpunkt der Markgrafen von Baden bildeten. Die damit einhergehende, grundlegende Neuorientierung der markgräflichen Herrschaft bahnte sich kurz nach dem Tod Hermanns V. an. Das deutlichste Zeichen dieser politischen Neuorientierung war sicher die ab 1243 fassbar werdende Gründung des Zisterzienserinnenklosters Lichtenthal und dessen Einrichtung als neues Hauskloster der Markgrafen von Baden mit der markgräflichen Grablege. Lichtenthal löste damit das ältere Hauskloster in Backnang ab, womit das religiöse Zentrum der Familie vom Neckar an den Oberrhein verschoben wurde. Als die Witwe Markgraf Hermanns V. dessen zunächst in Backnang beigesetzten Leichnam 1248 nach Lichtenthal umbetten ließ, begründete dies eine Begräbnistradition, die von den Markgrafen über 150 Jahre fortgeführt wurde. Neben Irmgard trat in Lichtenthal vor allem ihr Sohn Rudolf als zweiter und gewissermaßen eigentlicher Gründer der dortigen Hausgrablege hervor, denn Rudolf stiftete kurz vor seinem Tod jene Kapelle, die von der markgräflichen Familie bis ins 15. Jahrhundert hinein als Grablege genutzt wurde ${ }^{85}$. Die Wahl des Klosterstandorts in der Nähe des namengebenden Sitzes in Baden darf rückschauend als ausgesprochen zukunftsweisende Entscheidung gewertet werden. Denn der Herrschaftsschwerpunkt der Markgrafen von Baden verlagerte sich seit der Zeit Markgraf Rudolfs I. zunehmend vom mittleren Neckar an den Oberrhein und damit in das Kerngebiet des späteren, neuzeitlichen Territorialstaates der Markgrafschaft, während am mittleren Neckar die Grafen von Württemberg in ehemals markgräfliche Positionen einrückten.

${ }^{84}$ Es handelt sich um die Ehen zwischen dem Grafen Ulrich I. von Württemberg und Markgraf Hermanns V. Tochter Mathilde sowie zwischen dem Grafen Eberhard I. und Irmgard, einer Tochter Markgraf Rudolfs I.

${ }^{85}$ Siehe dazu und allgemein zur Bedeutung Markgraf Rudolfs I. für die Neuorientierung der markgräflichen Politik Krieg, Herrschaftsbildung (wie Anm. 9) S. 166-174. 